\title{
Synthesis and characterization of molecularly imprinted polymers for the remediation of PCBs and dioxins in aqueous environments
}

\author{
Phumile Sikiti, Titus AM Msagati, Bhekie B Mamba and Ajay K Mishra
}

\begin{abstract}
This paper, reports on the approach devised to remediate water sources contaminated with PCBs and dioxins. The approach reported is based on the synthesis of highly selective molecularly imprinted polymers (MIPs). The paper elaborates the materials, procedures and protocols devised and followed for the synthesis of MIPs. The characterization of the synthesized MIPs and NIPs were performed using a number of techniques, such as FTIR, SEM, etc. The FTIR results show a broad $\mathrm{OH}$ stretching vibration peaks associated with methacrylic acid carboxylic group (COOH). at $3710 \mathrm{~cm}^{-1}$ for NIP and $3588 \mathrm{~cm}^{-1}$ for MIP, $-\mathrm{CH}_{2}$ stretching peak at $2953 \mathrm{~cm}^{-1}$ for NIP, peaks due to the presence of methylene group in both MAA and EDMA appearing at $2951 \mathrm{~cm}^{-1}$ for MIP. The carbonyl group C $=\mathrm{O}$ stretching peak was observed in both MIP and NIP at $1721 \mathrm{~cm}^{-1}$ and this might have originated from MAA and EDMA respectively in both MIP and NIP. Weak combination bands from $1637 \mathrm{~cm}^{-1}$ to $1249 \mathrm{~cm}^{-1}$ and sharp bands at $1143 \mathrm{~cm}^{-1}$ specifically on MIPs spectra indicated the presence of aromatic ring of the template. The surface area of MIP was found to be $74.0010 \mathrm{~m}^{2} / \mathrm{g}$, thus larger than that for NIP which was $58.6519 \mathrm{~m}^{2} / \mathrm{g}$ due to the presence of cavities on MIPs. The fit of the Langmuir model was found to be $r^{2}=0.5842$ while Freundlich model were found to be $r^{2}=0.3241$, signifying that better correlation was with Langmuir than Freundlich.
\end{abstract}

\section{Introduction}

Remediation of persistent organic pollutants (PoPs) at ultra-trace is always mandatory due to the fact that, many of the pollutants belonging to this class of compounds and which are found in the environments are toxic even at minute concentrations. There are many remediation methods and techniques for PoPs, but the majority are based on absorption. In the past, activated carbon emerged as a powerful method for remediation of pollutants from aqueous media [1]. But this technique is known to be inadequate to remediate many organic pollutants at low concentration levels [2]. Moreover, activated carbon applications are restricted due to its high cost especially where high quality activated carbon is needed.

Therefore in order to reduce the associated costs, many attempts have been made to find inexpensive remediation techniques for PoPs. Among the methods that were found

\footnotetext{
* Correspondence: tmsagati@uj.ac.za

Department of Applied Chemistry, Faculty of Science, University of Johannesburg, Doornfontein Campus, Johannesburg 2028, South Africa
}

to be attractive in terms of cost effectiveness is the use of silicates minerals such as zeolites. However, zeolites have got one limitation in that, they show low efficiency in the remediation of persistent organic pollutants [3]. Membrane techniques such as reverse osmosis have shown high capability and efficiency in terms of removing PoPs from aqueous media, but like high grade activated carbon, reverse osmosis is known to be expensive in terms of costs of operation and manufacturing [2].

The use of carbon nanotubes (CNTs) on the other hand have shown potential as remediation technologies for PoPs in water, but the costs associated with their production is a limiting factor [4]. The dioxins group of compounds comprise of 75 different chemical class of polychlorinated dibenzo-p-dioxin (PCDDs), and 135 polychlorinated dibenzofurans (PCDFs). They are known to enter into the environmental matrices from sources such as chemical and pesticides manufacturing industries, pulp and paper bleaching industries, burning of household's trash, forest fires and burning of industrial and medical waste products [5]. While, polyaromatic biphenyls (PCBs) 
are comprised of 209 different chemical class of compounds, which were firstly produced due to their chemical stability, hence finding use in transformer capacitors, paints, printing inks and also in many other industrial applications [6]. These compounds also enter into the environment through the accidental spills and leaks during the transport of the chemicals or from the leaks on fires in transformer capacitors or other product containing PCBs [7].

However, out of 75 different chemicals class of dioxins, only 7 of them, 10 out of 135 furans, and 12 of 209 PCBs, are known to be highly toxic [8]. In addition, because of their low water solubility, hydrophobic character and resistance to metabolic degradation, these persistent organic pollutants (dioxins, furans and PCBs) are found in wide range of biological samples (e.g. adipose tissues), where they tend to bio-accumulate through food web [9]. Exposure to these molecules in animals and humans cause an adverse health effects including mutagenicity, carcinogenicity, reproductive disorders, immune suppression, birth defects and they are also endocrine disrupters [10]. Thus, there is need to develop an effective, yet economical methods for the removal of dioxins and PCBs at low concentration levels in environmental waters. More importantly, in May 2004, the Stockholm Convection resolutions on persistent organic pollutants entered into force with the intention of reducing or ultimately eliminating these pollutants. However, South Africa as part of this Convection is legally obligated to abide by the objectives of the Treaty and is thus expected to support research on persistent organic pollutants [11]. One of the attractive technologies that show potential for the removal of PCBs and dioxins in water is the use of molecularly imprinted polymers (MIPs). The important features for MIPs that are attractive include stability, low cost, ease of preparation, versatility and resistance for a wide range of $\mathrm{pH}$ and temperature [12-14].

Molecularly imprinted polymers are man-made polymers with recognition binding sites which are able to bind a molecule of interest or its structural analogous from complex sample matrices $[15,16]$. The MIPs molecule is formed based on the polymerization of functional monomers and cross-linker molecules in a complementary shape around a template molecule. When the template is extracted or it is removed, it results into the formation of recognition binding sites in the polymer matrix, hence the template/target analyte of interests can be recognized from the complex environmental samples into that polymer matrix. There are several steps that are involved in preparation of molecularly imprinting. The first steps involve the generation of the template molecule which is to be mixed with functional monomer (units that finally react to form the polymer). These monomers have specific chemical functional groups such as carboxyl, hydroxyl, amino, or aromatic groups which can bind to the specific molecule covalently or non-covalently. In this work the non-covalently interaction will be adopted due to the suitable interaction between template and functional monomer. Another attractive feature for non-covalently protocol is that it easily conducted, avoiding the tedious synthesis of polymerization complex, and also great variety of functionality can be produced into molecularly imprinted by using non-covalently interaction methods. The second step in the MIPs synthesis involves the formation of polymerization assembly using excess of cross-linking agent to give a highly cross-linked, rigid, glassy polymer with functional groups fixed in a specific orientation around the template molecule. Then the embedded template is then chemically extracted from the polymer, creating a rigid imprint of the molecule inside the polymer matrix (Scheme 1).

The application of MIPs for removal of target analyte species or as sorbents has been reported for many organic compounds from different samples matrices, for example different MIPs for different analytes such as atrazine [17], Nitrophenol [18] sulfonylureas [19] have been reported. In addition to these, MIPs for remediation of dioxin have as well been reported [20]. Hence, the aim of this work was to synthesize and characterize molecularly imprinted polymers for the efficient remediation of polyaromatic biphenyl (PCBs) and dioxins from water samples.

\section{Experimental}

\section{Materials and methods}

Standards 2,3,7,8 TCDD and PCB-1 (2-monochlorobiphenyl), PCB-28 (2,4,4-tri-chlorobiphenyl), PCB-101 (2,2, 4,5,5-pentachlorobiphenyl) and PCBs-77 (3,3',4,5' -tetrachlorobiphenyl), and the organic solvents (AR grade) were purchased from Riedel-de-Haën (Seelze-Hannover, Germany). Pesticide quality solvents (n-hexane and chloroform) were purchased from Panreac (Barcelona, Spain). Chemicals for the synthesis of molecularly imprinted polymers (MIPs), which include, functional monomer methacrylic acids (100 g, 98\% purity), porogen solvent chloroform anhydrous (1 L, GC grade 99\%), Standard of dioxin (2,3,7,8 TCDD) (1 mL, 97.5\% purity) which was used as a template, 1,1'- Azobis (cylohexanecarbonitrile) (25 g, 98\%) used as an initiator, cross linker ethylene glycol dimethacrylate ( $25 \mathrm{~g}, 98 \%)$, acetic acids (2.5 L, 100\%), methanol (1 L, 99.8\%), were all purchased from Sigma-Aldrich (St Louis, MO, USA). Nitrogen gas for purge was purchased from AFROX (Johannesburg, South Africa).

\section{Preparation of standards}

PCBs stock solutions (1000 mg/L) and 2,3,7,8-tetrachlorodibenzo-p-dioxin $(1 \mu \mathrm{g} / \mathrm{L})$ were prepared in hexane and were stored at $4^{\circ} \mathrm{C}$ in a refrigerator. Fresh working 


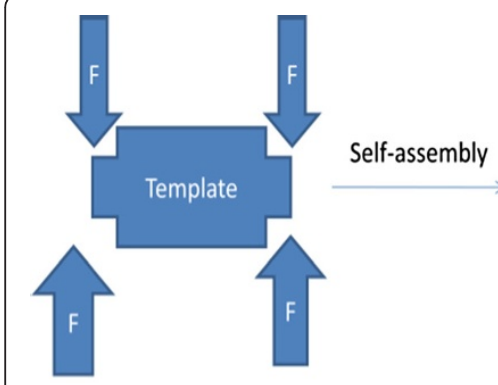

1)

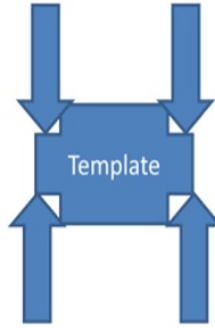

2)

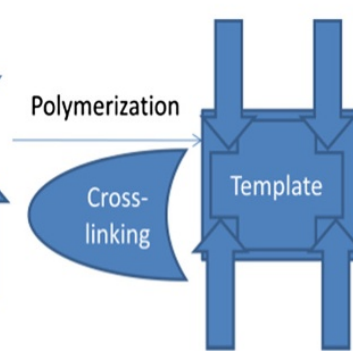

3)

4)

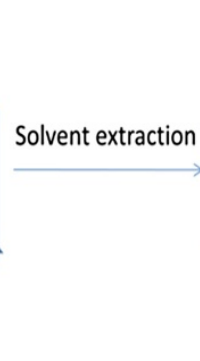

5)

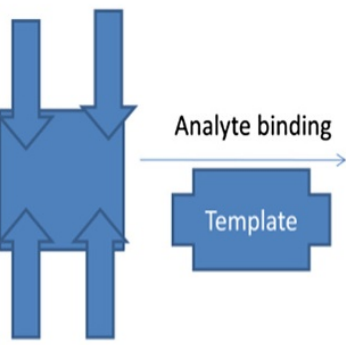

6)

7)

Scheme 1 Schematic representation of molecularly imprinted polymer synthesis using non-covalently interaction. Where 1) $f=$ functional monomer mixed with template followed by self-assembly by heating process. 2) Interaction between the template and functional monomer. 3 ) Polymerization and addition of cross-linking to get mechanically stabilize the polymer matrix - 4) followed by solvent extraction to remove template at step 5). At step 6) polymer matrix that complementary in shape, size. 7) The binding of analyte.

solutions were prepared daily by appropriate serial dilution of the stock solution with hexane as a solvent.

\section{Preparation of molecularly imprinted polymers and non-imprinted polymers}

In this work, molecularly imprinted polymers were synthesized via a bulk polymerization method, with 2,3, 7,8-tetrachlorodibenzo-p-dioxin as a template molecule, methacrylic acid (MAA) as a functional monomer, ethylene glycol dimethacrylate (EDMA) as a cross-linker, chloroform as porogenic solvent and 1,1' -azobis(cylohexanecarbonitrile) $(\mathrm{ABCHC})$ as an initiator.

In the procedure, different molar ratios of template to the functional monomer were prepared in order to get the best working molar ratio which was found to be 1:6. Therefore $1 \mathrm{mmol}$ of 2,3,7,8-tetrachlorodibenzo-p-dioxin and MAA were dissolved in $6 \mathrm{~mL}$ of chloroform in a flask, and the mixture was left to stand for few minutes for prearrangement and then $32 \mathrm{mmol}$ of EDMA and $0.103 \mathrm{~g}$ ABCHC were added. The prepared sample mixture was then degassed and purged with nitrogen before being sealed with a septum. The polymerization was carried out at $60^{\circ} \mathrm{C}$ for $24 \mathrm{hrs}$ in a thermostated water bath. The obtained polymers were mechanically grounded into powders, followed by washing using a mixture of methanol and acetic acid (9:1, v/v) in a Soxhlet apparatus to remove the template molecule. The powders with particle sizes ranging from 56 to $74 \mu \mathrm{m}$ were selected, washed, dried and stored in a desiccator. In a contrast experiment, the control polymer or the non-imprinted polymers (NIPs) were prepared using a similar procedure but without the addition of the template molecule, 2,3,7,8tetrachlorodibenzo-p-dioxin.

\section{Instrumentation}

The gas chromatograph model 7890A series (Agilent Technologies, Inc., Wilmington, Delaware, USA) coupled to a LECO Pegasus 4D time of flight mass spectrometer were used for all separations and detection of PCBs and dioxin extracts. Restek columns; Rxi-5Sil MS (30 m × $0.25 \mathrm{~mm}$ i.d $\times 0.25 \mu \mathrm{m}$ film thickness) primary column and Rtx-200 (0.69 m $0.18 \mathrm{~mm}$ i.d $\times$ 0.1B film thickness) secondary column were used for all separations. The flow rate of the carrier gas (helium) was $1.5 \mathrm{~mL} / \mathrm{min}$ and the injection type 100:1 split with volume of $2 \mu \mathrm{L}$. The injection temperature was set at $250^{\circ} \mathrm{C}$ and the oven temperature was programmed as follows: $35^{\circ} \mathrm{C}$ held for 0.25 minutes; ramped from $35^{\circ} \mathrm{C}-120^{\circ} \mathrm{C}$ at $60^{\circ} \mathrm{C} /$ minute, then $120^{\circ} \mathrm{C}-220^{\circ} \mathrm{C}$ at $80^{\circ} \mathrm{C} /$ minute.

The mass spectrometry conditions were set as follows: Ionization: Electron ionization at $-70 \mathrm{eV}$; source temperature: $180^{\circ} \mathrm{C}$; stored mass range: $47-350 \mathrm{um}$; acquisition rate: 20 spectra/second; detector voltage: $-1500 \mathrm{~V}$. For spectroscopic characterization, a Thermo Scientific (Nicolet IS 10) Fourier transform Infrared (FT-IR) was employed while thermogravimetric analysis was done using a Perkin Elmer TGA 4000. The surface morphology was analyzed by using scanning electron microscopy (SEM) from TESCAN WIRSAM scientific and precision equipment (PTY) LTD. The surface and porosity analysis of the synthesized polymers were performed using Brunauer, Emmett, Teller (BET) Micromeritics ASAP 2020 surface and porosity analyzer.

\section{Rebinding studies for PCB-77 compound}

For the preparation of a calibration curve of PCB-77, solutions of standards $(50-250 \mu \mathrm{g} / \mathrm{L})$ were prepared by dilution of $1000 \mu \mathrm{g} / \mathrm{L}$ solution in hexane. Then in another separate experiment, $3 \mathrm{~mL}$ of $(0.3-10 \mu \mathrm{g} / \mathrm{L})$ of PCB-77 in which $3 \mathrm{mg}$ of MIP and NIP were added separate vials. The solutions were then sealed and shaken in a shaker at $25^{\circ} \mathrm{C}$ for $24 \mathrm{hrs}$. The suspension was then filtered using $0.22 \mu \mathrm{m}$ PVDF filter. The concentration of the filtrate was 


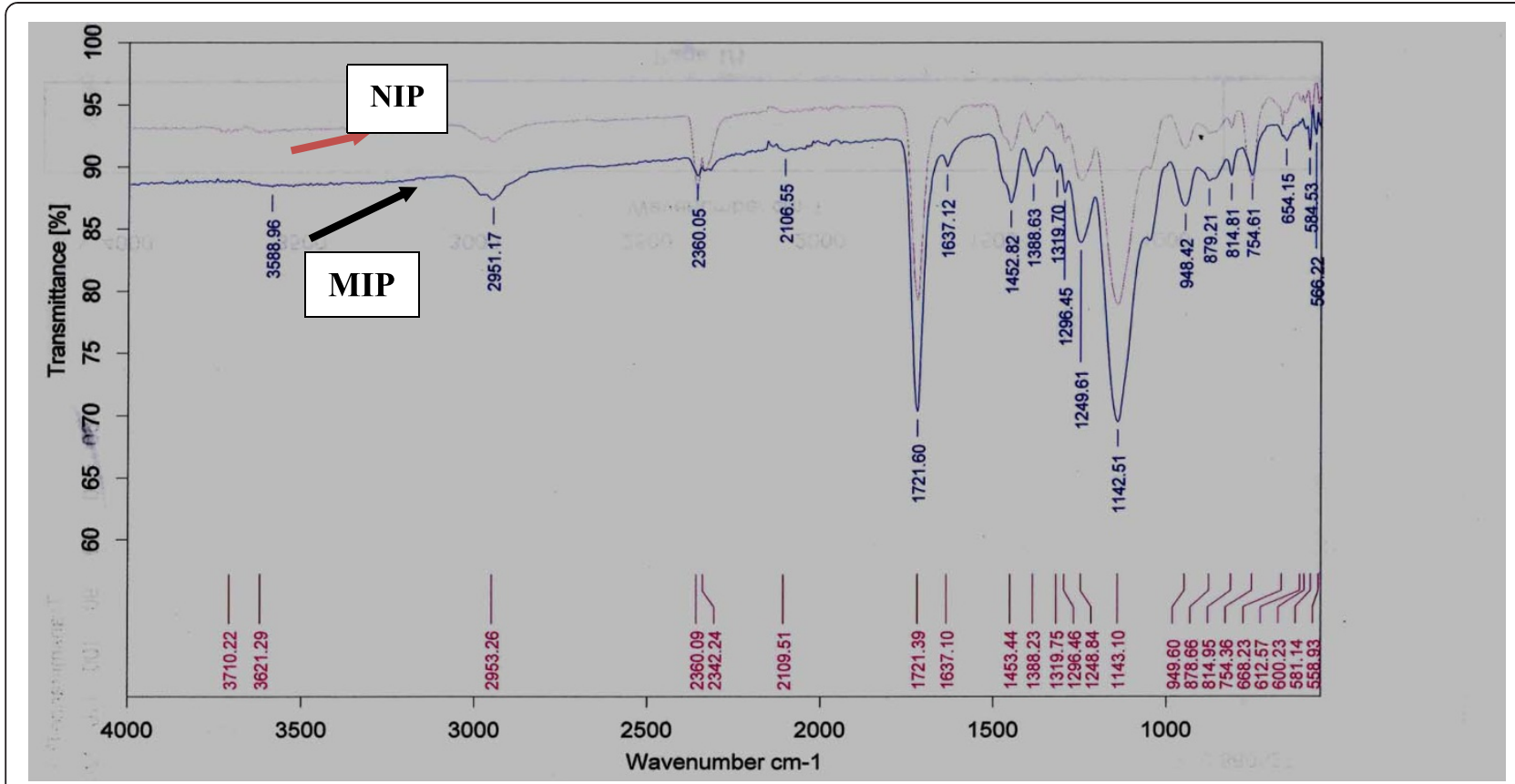

Figure 1 FTIR spectra of NIP and MIP.

analyzed by using GC-TOF-MS. The amount of analyte bound to the MIP was calculated using Equation 1.

$$
\mathrm{q}_{\mathrm{e}}=\mathrm{V} \frac{(\text { Co-Ce })}{\mathrm{m}}
$$

Where $\mathrm{q}_{\mathrm{e}}$ is the amount of analyte adsorbed in an adsorbent (MIP or NIP), V is the volume of the solution, $\mathrm{C}_{\mathrm{O}}$ is the initial concentration and $\mathrm{C}_{\mathrm{e}}$ is the concentration of target analyte at equilibrium or after adsorption.

\section{Results and discussion}

FTIR characterization of MIPs and NIPs

Molecularly imprinted polymers (MIPs) and non imprinted polymers (NIPs) were prepared via bulk polymerization method using a non-covalent approach. The interaction between functional monomer and template molecule provides the affinity and high selectivity of MIP. In this study FTIR in Figure 1 was used to confirm peaks after co-polymerization had been done. The FTIR results show a broad $\mathrm{OH}$ stretching vibration peak at $3710 \mathrm{~cm}^{-1}$ for
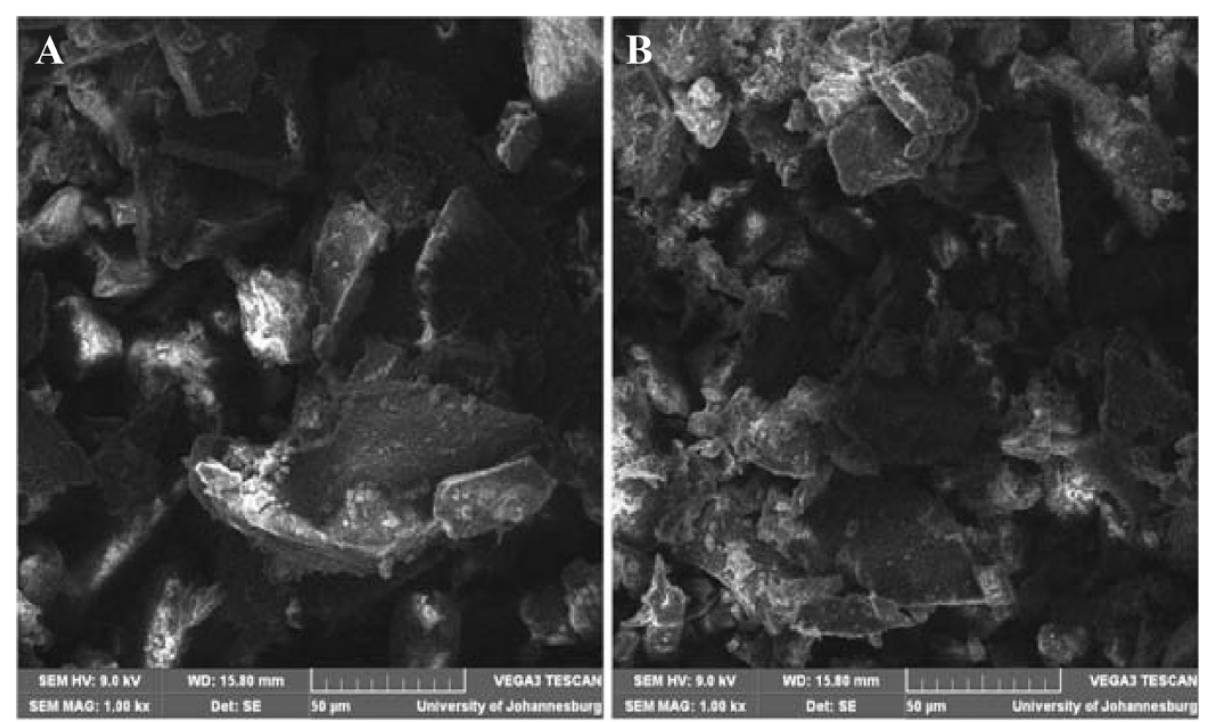

Figure 2 SEM micrograph images of A: = MIP and B: = NIP. 

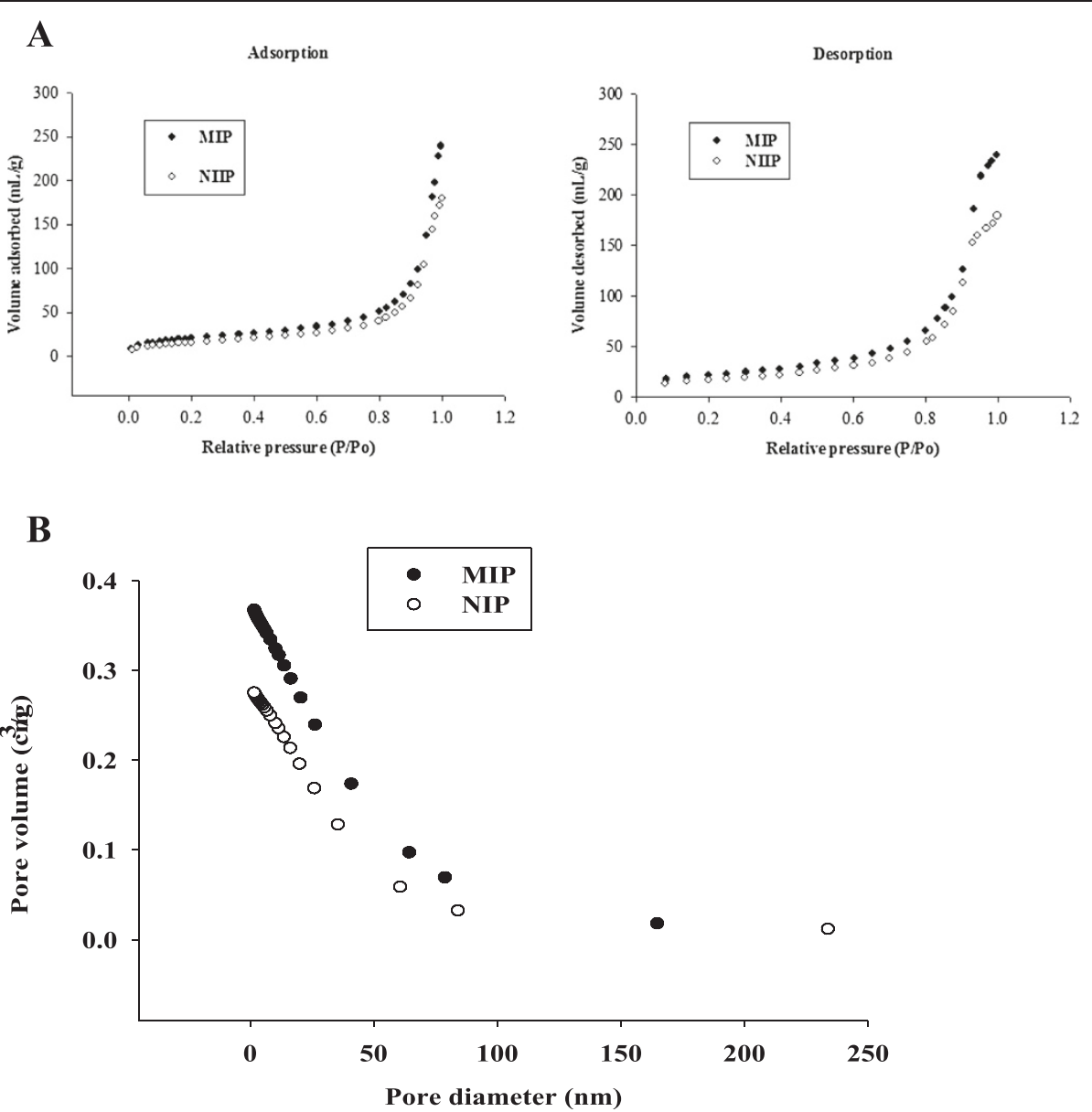

Figure 3 HPLC glass vial containing an insert. A. Adsorption of MIP and NIP, and desorption of MIP and NIP. B. Variation of pore volume versus pore diameter for MIP and NIP.

NIP and $3588 \mathrm{~cm}^{-1}$ for MIP. These peaks can be associated with methacrylic acid carboxylic group $(\mathrm{COOH})$. The $-\mathrm{CH}_{2}$ stretching peak was also observed at $2953 \mathrm{~cm}^{-1}$ for NIP while the peak appearing at $2951 \mathrm{~cm}^{-1}$ can be attributed for MIP, and this can be due to the presence of methylene group in both MAA and EDMA. The carbonyl group $\mathrm{C}=\mathrm{O}$ stretching peak was observed in both MIP and NIP at $1721 \mathrm{~cm}^{-1}$ and this might have originated from MAA and EDMA respectively in both MIP and NIP. Weak combination bands from $1637 \mathrm{~cm}^{-1}$ to $1249 \mathrm{~cm}^{-1}$ and sharp bands at $1143 \mathrm{~cm}^{-1}$ specifically on MIPs spectra indicate the presence of aromatic ring of the template.

\section{SEM characterization of both MIPs and NIPs}

The surface morphology and the particle size of both MIP and NIP were analyzed using scanning electron microscopy (SEM). The results indicates that the nature of the particles obtained after preparation of polymers by bulk polymerization were all found to be more irregular Figure $2 \mathrm{~A}$ and $\mathrm{B}$. The control polymer (NIP) was observed to have smoother surface than the MIP, while the MIP after the template removal on the other side had rough surfaces (Figure 2) which can be attributed to the formation of cavities during the synthesis process. It has been reported that the roughness of MIP particles can lead to high surface area than that of NIP and thus MIP can adsorb analytes of interest much better than the NIP.

\section{BET characterization of the imprinted (MIPs) and the non-imprinted (NIPs) polymers}

The adsorption-desorption isotherms of nitrogen $\left(\mathrm{N}_{2}\right)$ at $75.6 \mathrm{~K}$ on the MIPs and NIPs are shown in Figure 3A and $\mathrm{B}$ which show the relationship between the amount of nitrogen adsorbed relative to pressure. The volume adsorbed of MIP and NIP increased with relative pressure

Table 1 Surface area, pore volume, and pore size of MIP and NIP

\begin{tabular}{lccc}
\hline Polymer type & $\begin{array}{c}\text { Surface area } \\
\left(\mathbf{m}^{\mathbf{2}} \mathbf{/ g}\right)\end{array}$ & $\begin{array}{c}\text { Pore volume } \\
\left(\mathbf{c m}^{\mathbf{3}} \mathbf{/ g}\right)\end{array}$ & $\begin{array}{c}\text { Pore } \\
\text { size }(\AA)\end{array}$ \\
\hline MIP & 74.00 & 0.3704 & 200.2 \\
NIP & 59.00 & 0.2777 & 189.3 \\
\hline
\end{tabular}




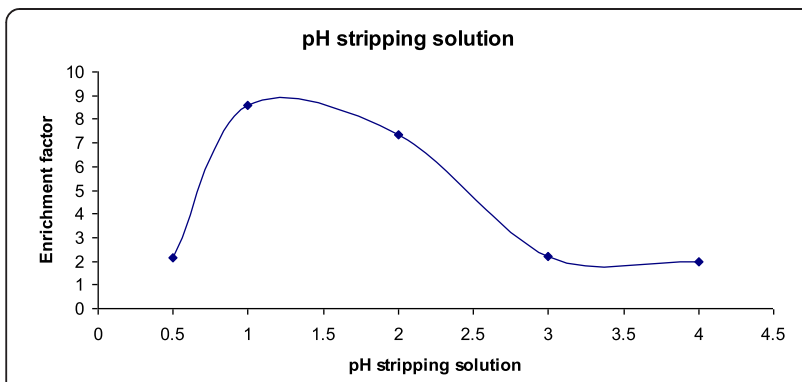

Figure 4 Acceptor pH optimisation for $1 \mathrm{mg} / \mathrm{L}$ carbofuran; membrane, $5 \%$ TOPO in isooctan, Sample $\mathrm{pH}=11$, extraction time $=30$ minutes and stirring speed of $300 \mathrm{rpm}$.

followed by a sudden increase in nitrogen uptake at a relative pressure from $\mathrm{P} / \mathrm{P}_{\mathrm{O}}<0.8$ and this might be due to capillary condensation. However, both MIP and NIP adsorbs and desorbed. The surface area of MIP was found to be $74.0010 \mathrm{~m}^{2} / \mathrm{g}$, thus larger than that for NIP which was $58.6519 \mathrm{~m}^{2} / \mathrm{g}$ (Table 1). This observation simply means that MIP had larger surface area than that of NIP due to the presence of cavities on MIPs. Moreover, the pore volume and pore size for MIP were also found to be larger than that of NIP. In this case the larger the surface area the larger the pore volume and pore size. This may be due to the presence template molecule, such that after its removal from the polymer, it left rough particles of the MIP with higher surface area.

The pore size distribution was investigated by observing the variation of collective pore volume with pore diameter as shown in Figure 4. The pore size ranged between 2-
$50 \mathrm{~nm}$ which indicated that the pores formed in both MIP and NIP are mesopores with few macropores above $50 \mathrm{~nm}$ in both MIP and MIP.

\section{TGA characterization of the imprinted (MIPs) and the} control (NIPs) polymers

The TGA plots of MIP and NIP are shown in Figure 5, whereby MIP plots show dehydration at temperatures of about $95^{\circ} \mathrm{C}$ to $100^{\circ} \mathrm{C}$, while NIP dehydration is at about $50^{\circ} \mathrm{C}$ to $100^{\circ} \mathrm{C}$. MIPs decompose gradually until $300^{\circ} \mathrm{C}$ then show stability from $400^{\circ} \mathrm{C}$ to $700^{\circ} \mathrm{C}$. While NIPs a change is observed at about $350^{\circ} \mathrm{C}$ to $500^{\circ} \mathrm{C}$ and remain stable up to $800^{\circ} \mathrm{C}$.

\section{Rebinding studies of PCBs-77}

A series of standard solutions of PCB-77 ranging from 100 to $250 \mu \mathrm{g} / \mathrm{L}$ were prepared by serial dilution from a stock solution of $1000 \mu \mathrm{g} / \mathrm{L}$. The linearity was shown from the standard curve by the line of best fit with $r^{2}=0.9995$ for the equation, $y=+715.208 x-10333.9$ as obtained from the graph. In addition the prepared concentrations were used for the binding studies for both the synthesized imprinted polymers (MIPs) and the control polymers (NIPs) whereby the binding was performed using solutions with concentrations ranging from 0.3 to $10 \mu \mathrm{g} / \mathrm{L}$.

However, in order to find the binding amount adsorbed versus initial concentrations were plotted. The amount adsorbed is important parameter, as that gives a measure of the amount of PCBs in the adsorbent.

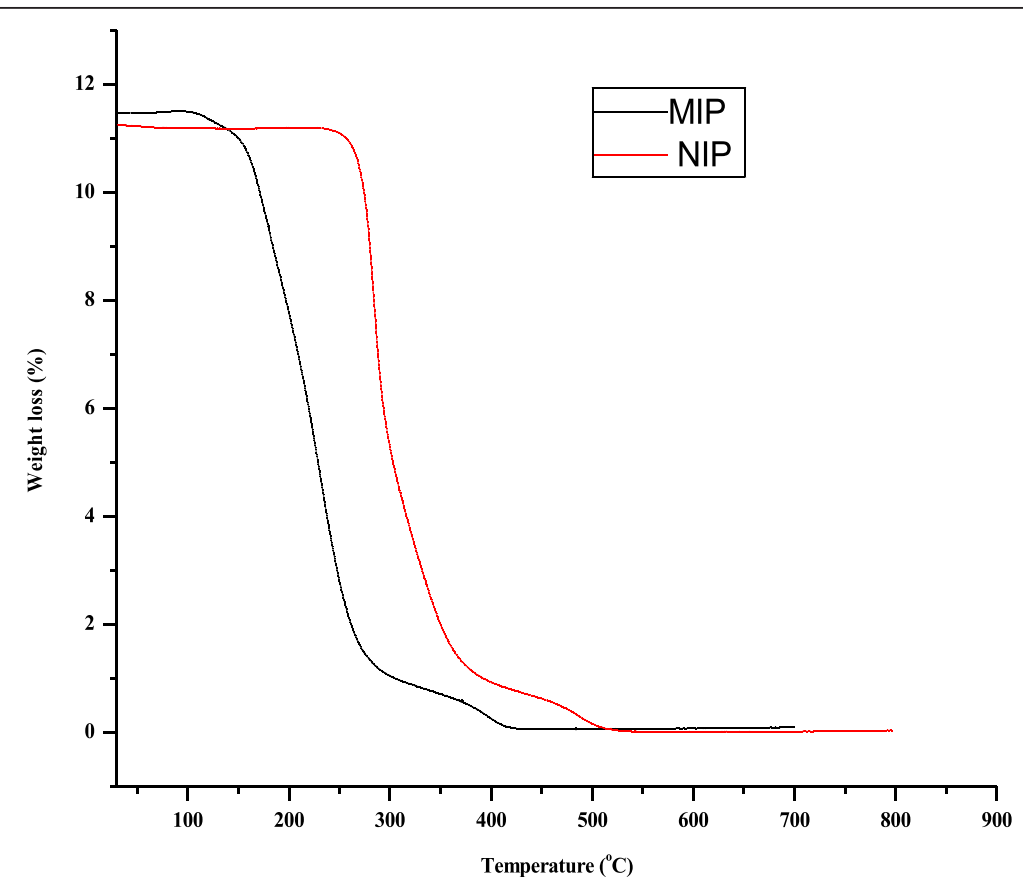

Figure 5 TGA decomposition curves of the imprinted (MIPs) and non-imprinted (NIPs) polymers. 


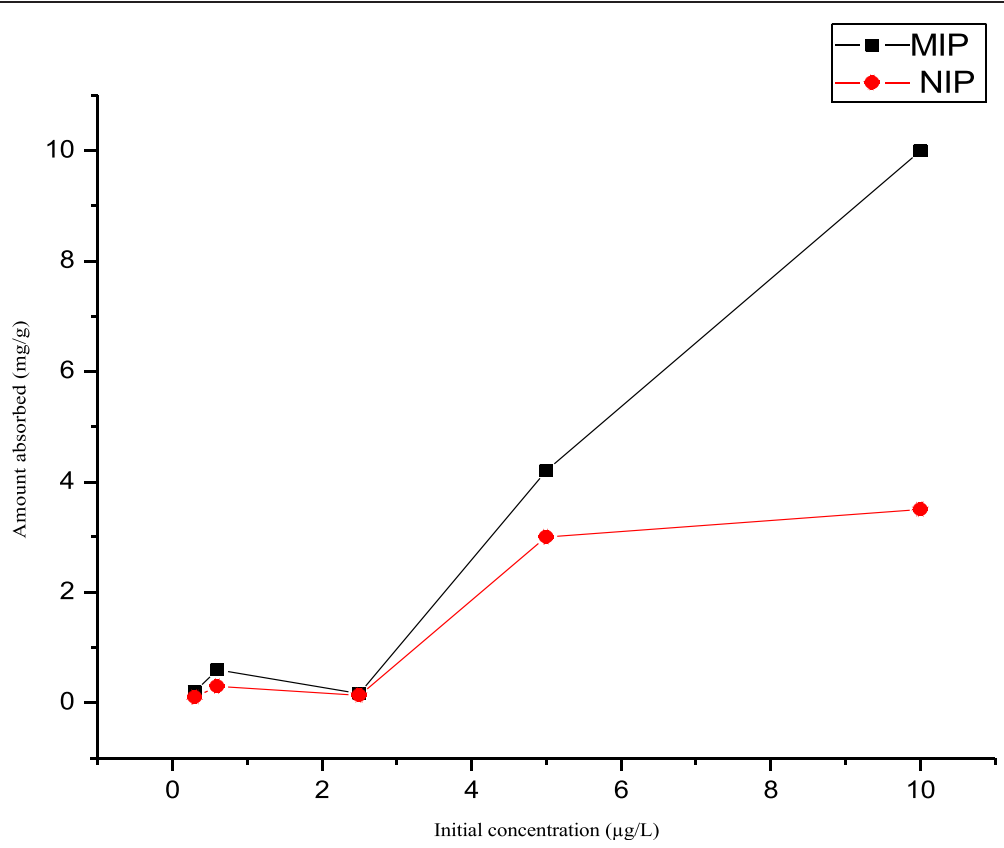

Figure 6 Comparison of adsorbed isotherm towards MIPs and NIPs.

In this study binding were carried out by varying the initial concentration of the 0.3 to $10 \mu \mathrm{g} / \mathrm{L}$ keeping other parameters such as volume of PCBs $(5 \mathrm{~mL})$ constant and mass of MIP or NIP (5 mg). The maximum binding capacity for PCB-77 in MIP was found to be $10 \mathrm{mg} / \mathrm{g}$ and for NIP was $3.5 \mathrm{mg} / \mathrm{g}$. Figure 6 shows the binding of MIP and NIP. The increase of initial concentration leads to the increase in the binding capacity and at the MIP show a higher analyte amount that was adsorbed than that for NIP. This is because NIP binds PCB-77 only at the surface (physisorption process); while on the other MIP is binding PCB-77 using the cavities formed during polymerization (chemisorption) and the surface.

However the fit of the Langmuir model was found to be $r^{2}=0.5842$ while Freundlich model were found to be $r^{2}=0.3241$. That show better correlation of the present data with Langmuir than Freundlich.

\section{Conclusions}

The use of MIPs for the removal of specific compounds from loaded waters is advantageous due to the high level of selectivity the MIPs offer. Since the synthesis is simple and straightforward, the preparation of these polymers can be performed in the most economical possible way and the application of such polymers can result in high efficiency in removing pollutants from contaminated water sources. Another attractive feature is that, the technology does not need highly skilled personnel to synthesize these polymers as the procedure is simple and it doesn't involve many steps. However, the optimization of the absorption parameters and kinetics parameters is in progress, hence that will conclude if the binding is controlled by monolayer or multilayer surfaces of MIPs towards PCBs and dioxin. In addition, the selectivity using analogues structural which are known to be persistent organic pollutant in the environment is also needed to be done.

\section{Competing interests}

The authors declare that they have no competing interests.

\section{Authors' contributions}

PS, carried out all the labwork (experiments), TAMM, BBM, AKM took part in the design of the experiments, supervision of the project. All authors read and approved the final manuscript.

\section{Acknowledgements}

Financial support from NIC-Mintek is gratefully appreciated.

Received: 15 June 2013 Accepted: 21 April 2014

Published: 9 May 2014

\section{References}

1. Nur Azreen Bt F, Ahmmed Saadi I: Review study for activated carbon from palm shell used for treatment of waste water: Utility reaction and environment. Journal of Purity 2012, 1(5):252-266.

2. Li DQ, Ma M: Inclusion chemistry to water purification technology. Journal of Chemistry Technology 1999, 35:31-37.

3. Salipira KL, Mamba BB, Krause RW, Malefetse TJ, Durbach SH: Cyclodextrin polyurethanes polymerised with carbon nanotubes for the removal of organic pollutants in water. Water SA 2008, 34(1):113-118.

4. Long R, Yang R: Carbon nanotubes as superior sorbent for dioxin removal. Journal of American Chemistry Society 2001, 123:2058-2058.

5. Im SH, Kannan KJ, Giesy JP, Matsuda M, Wakimoto T: Concentrations and profiles of polychlorinated dibenzo-p-dioxins and dibenzofurans in soils from Korea. J Environ Sci Technol 2002, 36(17):3700-3705.

6. US-EPA: PCBs. Cancer dose-response assessment and application to environmental mixtures. Washington, DC, USA: National Center for Environmental Assessment, 
Office of Research and Development, U.S. Environmental Protection Agency; 1996

7. ATSDR (Agency for Toxic Substances and Disease Registry): Toxicological profile for polychlorinated biphenyls (PCBS); division of toxicology/toxicology information branch 1600 Clifton road NE E-29 Atlanta Georgia. 4770 Buford Hwy NE: CDC Publication; 2000:30333. http:/www.atsdr.cdc.gov/substances/ toxsubstance.asp?toxid=26, accessed, 15-06-2013.

8. US-EPA: Bioaccumulation testing and interpretation for the purpose of sediment quality assessment and status and needs: EPA-823-R-00-001. Washington, DC: U.S. Environmental Protection Agency; 2000.

9. Voogt D, Wells DE, Reutergardh L, Brinkman UAT: International. Journal of Environmental Analytical Chemistry 1990, 40:1.

10. Van den Berg M, Birnbaum LS, Denison M: World health organization re-evaluation of human and mammalian toxic equivalency factors for dioxins and dioxin-like compounds. Journal of Toxicology Science 2006, 93(2):223-241.

11. Nieuwoudt C, Quinn LP, Pieters R, Jordaan I, Visser M, Kylin H, Borgen AR Dioxin-like chemicals in soil and sediment from residential and industrial areas in central South Africa. Chemosphere 2009, 76(6):774-83.

12. Haup K: Imprinted polymers-Tailor-made mimics of antibodies and receptors. Chemistry Communication 2003, 2:171-178.

13. Rahiminejad M, Shahtaheri SJ, Ganjali MR, Forushani AR, Golbabaei F: Molecurly impinted solis phase extraction for trace analysis of diazanon in drinking water. Iranian Journal Environmental Health Science Engineering 2009, 6(2):97-106.

14. Scorrano S, Mergola L, Del Sole R, Vasapollo G: Synthesis of molecularly imprinted polymers for amino acid derivates by using different functional monomers. Int J Mol Sci 2011, 12(3):1735-43.

15. Mayes AG, Whitcombe MJ: Synthetic strategies for the generation of molecularly imprinted organic polymers. Journal of Advanced Drug Delivery Revision 2005, 57:1742-1778.

16. Tamayo FG, Turiel E, Mart'in-Esteban A: Molecularly imprinted polymers for solid-phase extraction and solid-phase microextraction: recent developments and future trends. J Chromatogr A 2007, 1152:32-40.

17. Koohpaei AR, Shahtaheri SJ, Ganjali MR, Rahimi-Froushani A, Golbabaei F: Molecular imprinted solid phase extraction for trace level determination of atrazine in environmental samples. Iranian Journal Environmental Health Science Engineering 2008, 5:283-296.

18. Masque N, Marce RM, Borrull F, Cormack PAG, Sherrington DC: Synthesis and evaluation of a molecularly imprinted polymer for selective on line solid-phase extraction of 4-nitrophenol from environmental water. J Anal Chem 2000, 72:4122-4126.

19. Zhu QZ, Degelmann P, Niessner R, Knopp D: Selective trace analysis of sulfonylurea herbicides in water and soil samples based on solid-phase extraction using a molecularly imprinted polymer. J Environ Sci Technol 2002, 36:5411-5420.

20. Cosimino M, Rosaria AP, Giuseppe C, Vito S, Magda B: Synthesis of a molecularly imprinted polymer for dioxin. Journal of Sensors 2006, 6:915-924.

doi:10.1186/2052-336X-12-82

Cite this article as: Sikiti et al:: Synthesis and characterization of molecularly imprinted polymers for the remediation of PCBs and dioxins in aqueous environments. Journal of Environmental Health Science \& Engineering 2014 12:82.

\section{Submit your next manuscript to BioMed Central and take full advantage of:}

- Convenient online submission

- Thorough peer review

- No space constraints or color figure charges

- Immediate publication on acceptance

- Inclusion in PubMed, CAS, Scopus and Google Scholar

- Research which is freely available for redistribution 